\title{
Implementação do serviço de enfermagem em Podiatria Clínica em unidade pública
}

\section{de saúde ambulatorial}

\author{
Implementation of the nursing service in podiatria clinic in public outpatient health unit \\ Implantación del servicio de enfermería en la clínica podiatria de la unidad pública de salud \\ ambulatoria
}

Recebido:22/04/2021 | Revisado: 01/05/2021 | Aceito: 04/05/2021 | Publicado: 19/05/2021

\author{
Ariane da Silva Pires \\ ORCID: https://orcid.org/0000-0003-1123-493X \\ Universidade do Estado do Rio de Janeiro, Brasil \\ E-mail: arianepiresuerj@gmail.com \\ Eugenio Fuentes Pérez Júnior \\ ORCID: https://orcid.org/0000-0003-4611-0443 \\ Universidade do Estado do Rio de Janeiro, Brasil \\ E-mail: eugenioperezjunior@gmail.com \\ Eliana Gomes Marques \\ ORCID: https://orcid.org/0000-0003-0832-4918 \\ Universidade do Estado do Amazonas, Brasil \\ E-mail: elianamg@edu.br \\ Suely Rodrigues Thuler \\ ORCID: https://orcid.org/0000-0002-7003-7293 \\ Universidade do Estado do Amazonas, Brasil \\ E-mail: contato@staycare.com.br \\ Vera Ligia Lellis Jacob \\ ORCID: https://orcid.org/0000-0001-8268-2751 \\ Universidade do Estado do Rio de Janeiro, Brasil \\ E-mail: veraligia@clinicaveraligia.com.br \\ Francisco Gleidson de Azevedo Gonçalves \\ ORCID: https://orcid.org/0000-0002-6468-8137 \\ Universidade do Estado do Rio de Janeiro, Brasil \\ E-mail: gleydy_fran@hotmail.com \\ Kelly Fernanda Assis Tavares \\ ORCID: https://orcid.org/0000-0003-2445-6208 \\ Universidade do Estado do Rio de Janeiro, Brasil \\ E-mail: kfassis@yahoo.com.br \\ Juliana Agra Santos \\ ORCID: https://orcid.org/0000-0001-7619-8533 \\ Universidade do Estado do Rio de Janeiro, Brasil \\ E-mail: juagrasantos@gmail.com \\ Vânia Lima Coutinho \\ ORCID: https://orcid.org/0000-0001-9300-3697 \\ Universidade do Estado do Rio de Janeiro, Brasil \\ E-mail:vlcoutto@hotmail.com \\ Ellen Marcia Peres \\ ORCID: https://orcid.org/0000-0003-4262-6987 \\ Universidade do Estado do Rio de Janeiro, Brasil \\ E-mail: ellenperes@globo.com \\ Alessandra Sant' Anna Nunes \\ ORCID: https://orcid.org/0000-0001-7435-2568 \\ Universidade do Estado do Rio de Janeiro, Brasil \\ E-mail: asantnunes@gmail.com
}

\section{Resumo}

Objetivo: descrever a implementação de serviço de Enfermagem em Podiatria Clínica em unidade pública de saúde do Estado do Rio de Janeiro. Método: pesquisa convergente assistencial descrevendo o processo de implementação do serviço. Resultados: a avaliação da implementação do serviço proporcionou a realização de um mil cento e oitenta e cinco atendimentos em três anos, contribuindo significativamente para diminuição de complicações e agravos nos pacientes com diabetes. Além disso, contribuiu com a formação profissional qualificada de sessenta e quatro enfermeiros que se habilitaram na área de Podiatria Clínica, na cidade do Rio de Janeiro, por meio do curso de extensão. Conclusão: a implementação do serviço de Podiatria Clínica contribuiu para a diminuição das complicações 
do pé diabético, além de proporcionar melhor qualidade do cuidado de enfermagem ofertado a clientela do Sistema Único de Saúde por meio de assistência especializada, além de contribuir na formação dos profissionais com interesse na área que buscavam por qualificação.

Palavras-chave: Podiatria; Cuidados de enfermagem; Prática avançada de enfermagem; Pé diabético; Tecnologias em saúde.

\begin{abstract}
Objective: to describe the implementation of a Nursing service in Podiatry Clinic in a public health unit in the State of Rio de Janeiro. Method: convergent care research describing the service implementation process. Results: the evaluation of the implementation of the service provided the performance of one thousand eighty-five visits in three years, contributing significantly to the reduction of complications and injuries in patients with diabetes. In addition, it contributed to the qualified professional training of sixty-four nurses who qualified in the podiatry a clinic area, in the city of Rio de Janeiro, through the extension course. Conclusion: the implementation of the Clinical Podiatry service contributed to the reduction of complications of diabetic foot, besides providing better quality of nursing care offered to the clientele of the Unified Health System through specialized care, besides contributing to the training of professionals with interest in the area seeking qualification.
\end{abstract}

Keywords: Podiatry; Nursing care; Advanced nursing practice; Diabetic foot; Health technologies.

\title{
Resumen
}

Objetivo: describir la implementación de un servicio de Enfermería en la Clínica podología en una unidad de salud pública en el estado de Río de Janeiro. Método: investigación de atención convergente que describe el proceso de implementación del servicio. Resultados: la evaluación de la implementación del servicio proporcionó el desempeño de mil ochenta y cinco visitas en tres años, contribuyendo significativamente a la reducción de complicaciones y lesiones en pacientes con diabetes. Además, contribuyó a la formación profesional cualificada de sesenta y cuatro enfermeras que calificaron en la podología un área clínica, en la ciudad de Río de Janeiro, a través del curso de extensión. Conclusión: la implantación del servicio de Podología Clínica contribuyó a la reducción de complicaciones del pie diabético, además de proporcionar una mejor calidad de la atención de enfermería ofrecida a la clientela del Sistema Unificado de Salud a través de la atención especializada, además de contribuir a la formación de profesionales con interés en la zona en busca de cualificación.

Palabras clave: Podología; Cuidado de enfermería; Práctica avanzada de enfermería; Pie diabético; Tecnologías de la salud.

\section{Introdução}

O objeto desta pesquisa trata da implementação de um serviço de enfermagem especializado na área de Podiatria Clínica como ferramenta para o cuidado qualificado de enfermagem a pacientes com diabetes. Esse objeto emergiu como consequência dos cuidados assistenciais prestados a partir de ações extensionistas que foram implementadas em um ambulatório de diabetes, em um complexo ambulatorial que pertence a uma universidade pública no Estado do Rio de Janeiro, através de dois projetos de extensão universitária intitulados "Podiatria Clínica: Formação e Cuidado Especializado em Enfermagem" e "Laserterapia de Baixa Intensidade na Prática Clínica de Enfermagem”. Esses projetos de extensão foram idealizados e implementados fundamentados na necessidade de ofertar atendimento de enfermagem especializado congregando o uso de tecnologias na assistência a pacientes portadores de patologias nos membros inferiores.

A alocação dos projetos no serviço de diabetes da referida instituição justifica-se pelo fato de nesse setor haver grande concentração de pacientes com distúrbios nos membros inferiores devido às complicações do diabetes, e portanto, maior demanda de cuidados especializados no que tange a prevenção, tratamento e reabilitação dos pacientes.

Ressalta-se que o Diabetes Melito (DM) e suas complicações estão se tornando rapidamente a principal causa de morbimortalidade no mundo. Prevê-se que, até 2040, haverá mais de 642 milhões de pessoas com diabetes no mundo ${ }^{1}$.

Pacientes com DM possuem taxa de incidência anual de úlceras plantares de $2 \%$, além do risco de $25 \%$ de desenvolvimento destas ao longo de suas vidas. $85 \%$ das amputações de membros inferiores em pacientes com DM são precedidas de úlceras, sendo os seus principais fatores de risco a neuropatia periférica (NP), as deformidades nos pés e os traumatismos ${ }^{2}$. 
As alterações de ordem neurológica e vascular em extremidades, provocadas pelo quadro DM, produzem distorções na anatomia e fisiologia normais dos pés. A alteração do trofismo muscular e da anatomia óssea dos pés provoca o surgimento de pontos de pressão, enquanto o ressecamento cutâneo prejudica a elasticidade protetora da pele e o prejuízo da circulação local torna a cicatrização mais lenta e ineficaz. Em conjunto, essas alterações aumentam o risco de úlceras nos pés, podendo evoluir para complicações mais graves, como infecções e amputações².

A doença arterial periférica (DAP), geralmente em combinação com traumas leves ou danos triviais, pode resultar em uma úlcera dolorosa, puramente isquêmica. Entretanto, a DAP e a neuropatia estão presentes, mais frequentemente, no mesmo paciente ${ }^{1}$.

É possível que a redução no fluxo sanguíneo na pele, devido à doença macrovascular, torne a vascularização mais sensível à oclusão durante períodos de elevada pressão biomecânica na pele. É improvável, no entanto, que a doença microvascular constitua uma causa direta da ulceração. A microangiopatia desempenha um papel importante na patogênese das úlceras do pé diabético; é um espessamento da membrana basal e o edema endotelial nos capilares, porém não causa bloqueio. Deve-se acrescentar que as artérias distais são responsáveis pelo suprimento arterial dos pododáctilos ${ }^{3}$.

A neuropatia periférica provoca desarranjos que prejudicam os mecanismos neuro-mio-osteo-articulares, juntamente com a perda da sensibilidade protetora (PSP) ocasionando um aumento do gasto energético para marcha e os pontos de hiperpressão plantar, deteriorando a qualidade deambulatória funcional e com riscos de ulceração, infecção e amputação ${ }^{4}$.

As úlceras plantares, nos pacientes diabéticos, são consideradas feridas muito complexas, sentenciando os pacientes a tratamentos arrastados por longos anos, que por diversas vezes podem não levar a uma resolução do seu problema e deixandoos ainda vulneráveis a infecções e amputações.

Além das úlceras plantares outra situação de complicação nos membros inferiores bastante recorrente na população que convive com o diabetes são as micoses, principalmente aquelas que acometem as unhas (onicomicose) e/ou na região interdigital (tínea pedis).

A micose é a infecção determinada por diversas espécies de fungos: dermatófitos, não dermatófitos e leveduras. A distribuição destes diferentes patógenos não é uniforme, e depende de vários fatores tais como clima, área geográfica e migração. Os agentes causais das onicomicoses dermatofíticas são os fungos do gênero Trichophyton e Epidermophyton, e excepcionalmente Microsporum. Nos últimos anos, os casos de onicomicoses não dermatofítica, aumentaram rapidamente. Os fatores que contribuem para a instalação de onicomicose podem ser divididos em: gênero, perturbações circulatórias periféricas, resistência diminuída, traumatismo e fatores de manutenção, como profissão, clima, disfunção hormonal ${ }^{5}$.

Mesmo com o grande avanço das práticas de saúde e conhecimento amplo das complicações dos membros inferiores em diabéticos, estas podem surgir na ocorrência de falhas na prevenção associadas aos fatores de risco. Devido a isso, deve-se ter o conhecimento de várias tecnologias que possam auxiliar na abordagem rápida e eficaz, a fim de evitar maiores riscos a esses pacientes tão vulneráveis. Nesse contexto, surge a necessidade de cuidados especializados dos pés através da Enfermagem em Podiatria Clínica congregando o uso de tecnologias em saúde como a laserterapia de baixa intensidade.

Neste contexto, elaborou-se como objetivo de estudo: descrever a implementação de um serviço de enfermagem especializado na área de Podiatria Clínica como ferramenta para o cuidado qualificado de enfermagem a pacientes com diabetes.

\section{Referencial Teórico}

A Podiatria, em nível mundial, originou-se da Quiropodia (especialidade médica), a qual envolve a melhora da saúde global do paciente, com foco na prevenção primária, diagnosticando e tratando problemas associados aos pés e tornozelos ${ }^{6}$. 
A primeira sociedade de Quiropodistas foi estabelecida em 1895 na cidade de Nova Iorque e em 1911 foi fundada a primeira escola de Quiropodia pelo médico e educador Dr. Maurice J. Lewi, conhecida anos depois como Faculdade de Nova Iorque de Medicina Podiátrica. Dr. Lewi ajudou a desenvolver a $1^{\text {a }}$ legislação que norteou a prática dos Quiropodistas e, as disciplinas e o programa de treinamento do $1^{\circ}$ curso de formação em Quiropodia, nesta escola ${ }^{6}$.

Historicamente, no Brasil, este cuidado que já era desenvolvido pelo Enfermeiro, sendo foi reconhecido como especialidade pelos órgãos competentes da época, pelo Decreto de Lei 10068, publicado na Secretaria de Estado da Educação e Saúde Pública, em 23 de março de 19397.

“§ $2 .^{\circ}$ - Os enfermeiros-pedicuras poderão instalar sala de trabalho, guarnecida com os móveis e instrumentos estritamente necessários à sua especialidade, e cuja abertura deverá ser autorizada pelo Serviço de Fiscalização do Exercício Profissional - artigo 10, inciso $2^{\circ}$ do decreto ${ }^{7 ”}$.

No âmbito da assistência em saúde, destaca-se a atuação da Enfermeira Podiatra Vera Ligia Lellis Jacob, pioneira na área da Podiatria Clínica Brasileira. Através de suas experiências clínicas em mais de 25 anos de assistência prestada a pacientes diabéticos com complicações nos pés e idosos com pé geriátrico, atuou no cuidado a essa população no "Ambulatório de Pé Diabético" do Hospital Estadual Brigadeiro, Hospital das Clínicas de São Paulo e posteriormente, em 2005, na Clínica Integrada de Prevenção e Tratamento dos Pés (CLIPÉS), atualmente, Clínica Vera Ligia.

A expansão da Podiatria Clínica Brasileira contou com o protagonista da Enfermeira Vera Ligia e de muitos outros profissionais com expertise na área e atuação profissional voltada para assistência dos pacientes com afecções nos membros inferiores que também contribuíram, sobremaneira, para o crescimento e a difusão da Podiatria Clínica no Brasil, como por exemplo, cita-se a Profa. Dra. Mônica Antar Gamba, enfermeira, docente da Universidade Federal de São Paulo e Especialista em Enfermagem em Dermatologia. A enfermeira e professora, no âmbito da assistência e ensino de enfermagem, enfocou especial atenção para a importância do cuidado de enfermagem com os pés, com olhar sensível e holístico ao indivíduo. Contribuindo ainda, com publicações de relevante importância acadêmico-científica na área.

No que se refere à formação, em 2007, ocorreu o primeiro Curso de Pós-graduação Lato Sensu (Especialização) de Enfermagem em Podiatria Clínica na Universidade Federal de São Paulo (UNIFESP) - Lato Sensu 364/2007, aprovado pelo Conselho Federal de Enfermagem sob o parecer ASJE nº 057/2005.

$\mathrm{O}$ referido curso foi idealizado pela Enfermeira Vera Ligia Lellis Jacob, que atuou como docente e na equipe de coordenação, juntamente com a Profa. Dra. Odete de Oliveira Monteiro. Entre os anos de 2007 a 2009 foram concluídas três turmas, com 52 egressos com formação pós-graduada em Podiatria Clínica. Em parceria com uma instituição privada de ensino a Enfermeira Vera Ligia, promoveu mais duas turmas em nível de pós-graduação (especialização) nos anos de 2012 a 2013. Os mencionados cursos tiveram carga horária de 550 horas (teórico-prático) e integralização em 18 meses letivos. Após a realização dessas cinco turmas de Especialização em Enfermagem em Podiatria Clínica, a especialidade permaneceu adormecida no país.

Nasce então em 2015 o Curso de Habilitação em Podiatria Clínica. Essa modalidade de formação promove qualificação profissional aos enfermeiros com interesse na área. Os Cursos de Habilitação em Podiatria Clínica, têm carga horária mínima de 180 horas, são de natureza teórico-prática, e oferecidos apenas para Graduados em Enfermagem.

A exemplo, esses cursos são ofertados nas cidades de: São Paulo, (desde 2015), Santa Catarina (desde 2016), Curitiba (desde 2017) e no Rio de Janeiro (desde 2018).

Em 2018, na região norte, na cidade de Manaus, na Universidade do Estado do Amazonas (UEA), sob coordenação das enfermeiras e professoras: Profa. Ms. Eliana Gomes Marques (Especialista em Enfermagem em Estomaterapia e Docente da UEA) e Profa. Ms. Suely Rodrigues Thuler (Especialista em Podiatria Clínica e Diretora da Stay Care) ressurgi então a 
formação pós-graduada, através do Curso de Especialização em Podiatria Clínica para Enfermeiros, (edital 076/2018 GR/UEA). A turma foi composta por 42 alunos, obteve carga horária de 452 horas, proposta de integralização em 18 meses letivos, cuja conclusão ocorreu em outubro de 2020.

Atualmente, na região sudeste do país, o curso de especialização, também se encontra aprovado na Faculdade de Enfermagem da Universidade do Estado do Rio de Janeiro.

Ressalta-se que o mercado de trabalho para o enfermeiro pós-graduado em Podiatria Clínica encontra-se em expansão tanto em nível nacional quanto internacional e como exemplo cita-se a atuação de um Enfermeiro Podiatra Brasileiro no Clube de Futebol Chinês (Enfermeiro Otávio Junior Rodrigues).

A Podiatria Clínica é uma especialidade da enfermagem que objetiva a saúde dos pés com ênfase no cuidado dos membros inferiores, levando em consideração, além da manutenção ou recuperação da saúde, alterações posturais e da biomecânica do movimento, com foco na reabilitação. Sendo assim, o Enfermeiro Podiatra ou Habilitado em Podiatria Clínica têm exímio papel na equipe multidisciplinar através de quatro pilares centrais: avaliação, prevenção, tratamento e reabilitação dos indivíduos para promoção da saúde e bem-estar.

Por se tratar de uma especialidade recente da enfermagem brasileira, ainda ocorrem desajustes acerca da titulação dos profissionais e uso equivocado do título de "Podiatra". Os concluintes dos cursos devem receber as menções de acordo com o tipo de curso realizado, no caso dos cursos de Habilitação com carga horária mínima de 180 horas, os concluintes poderão receber o título de "Habilitado em Podiatria Clínica", estando reservado o título de "Podiatra" apenas para os Enfermeiros com Pós-Graduação concluída na área de Podiatria Clínica, com carga horária mínima de 360 horas, conforme orientação do Ministério de Educação e Cultura (MEC).

\section{Metodologia}

Entende-se por método o caminho do pensamento e a prática exercida na abordagem da realidade. A metodologia de pesquisa inclui as concepções teóricas de abordagem, o conjunto de técnicas que possibilitam a construção da realidade e o potencial criativo do investigador. Além disto, ela deve apresentar um instrumental claro, coerente, elaborado e capaz de encaminhar os impasses teóricos para o desafio da prática ${ }^{8}$.

Para a pesquisa em tela, optou-se pelo método da Pesquisa Convergente Assistencial (PCA). O conceito de convergência na PCA é entendido como sendo o entrecruzamento de ações de assistência com as ações de pesquisa, encontro esse que proporciona possibilidades de leitura e descoberta de novos fenômenos ${ }^{9}$.

A PCA é um método de pesquisa que se envolvem com a transformação da realidade, incluindo algumas desigualdades sociais e econômicas, podem ter uma contribuição mais efetiva em países em desenvolvimento como o Brasil, e tem conquistado o reconhecimento de sua pertinência e contribuição ${ }^{10}$.

A implementação do serviço de Enfermagem em Podiatria Clínica ocorreu em uma unidade ambulatorial pública que oferece atenção secundária especializada. Esta unidade de saúde pertence a uma universidade pública do Rio de Janeiro, desde 1995, fomentada pelo Ministério da Saúde com a missão de ser espaço de formulação, implementação e avaliação de modelos em saúde para o fortalecimento do Sistema Único de Saúde, favorecer a integração docente assistencial e enfatizar práticas de saúde preventivas e resolutivas, estando inserida na rede de atenção à saúde do munícipio como, a qual oferta consultas e serviços ambulatoriais especializados, por meio do Sistema de Regulação (SISREG) atendendo as solicitações feitas pelas unidades de atenção básica e também por regulação interna ${ }^{11}$.

Neste complexo ambulatorial são disponibilizadas especialidades como: Alergia e Imunologia, Cardiologia, Geriatria, Cirurgia Geral, Cirurgia Plástica, Cirurgia Vascular, Clínicas Integras/ Especializadas, Disfunção Miccional, Endocrinologia, 
Estomaterapia, Gastroenterologia, Ginecologia, Medicina Integral, Neurologia, Pediatria, Podiatria Clínica, Pneumologia, Centro de Infusão, Reumatologia, Saúde do Homem, Saúde Mental, Urologia e DIP/APA ${ }^{11}$.

Inicialmente a proposta de construção do serviço teve como base a necessidade de atendimento especializado aos pacientes portadores de diabetes com complicações nos membros inferiores que necessitavam de atendimento de Enfermagem em Podiatria Clínica.

O ambulatório de diabetes, local de implementação, dispõe de equipe multidisciplinar de saúde, composta por profissionais médicos especializados em endocrinologia, equipe de enfermagem (técnicos e enfermeiros), nutrição, psicologia, assistência social e parceria com o serviço de cirurgia vascular. Além disso, conta com os residentes médicos, de enfermagem e equipe técnica e administrativa.

Em virtude de sua relevância clínica e da grande demanda de pacientes com complicações nos membros inferiores necessitando de cuidados especializados os projetos de extensão de "Podiatria Clínica" e "Laserterapia de Baixa Intensidade" conquistaram um espaço no referido ambulatório e instalaram um "Consultório de Enfermagem em Podiatria Clínica", no serviço de diabetes, pautado em duas linhas principais de trabalho: uma no âmbito da assistência especializada através da prestação de serviços atendo usuários do Sistema Único de Saúde (SUS), e a outra na formação promovendo cursos de capacitação profissional para profissionais (enfermeiros) com interesse na área de podiatria e tecnologias em saúde.

Após a obtenção do espaço físico, realizou-se pesquisas orçamentarias para a aquisição de materiais permanentes, instrumentais especializados e tecnologias que foram adquiridos através da captação de recursos de cursos autogeridos promovidos em nível de especialização e extensão. Sobre os materiais de consumo não específicos e descartáveis pactuou-se a obtenção por meio da própria instituição que também dispõe de rede de internet e sistema de prontuário eletrônico.

Sobre os recursos tecnológicos adquiridos, optou-se pela aquisição de dois aparelhos de laserterapia de baixa intensidade, um aparelho de ozonioterapia, um aparelho de emissão de luz UVC (que realiza desinfecção de calçados) e equipamentos/materiais para avaliação podopostural e confecção de palmilhas sob molde.

Em relação aos aspectos éticos está pesquisa foi aprovada pelo Comitê de Ética da instituição investigada sob CAAE: 16427419.3.0000.5259, conforme preconiza a Resolução no 466/2012, do Conselho Nacional de Saúde (CNS/MS) ${ }^{12}$.

\section{Resultados}

O Serviço de Podiatria Clínica foi implementado em abril de 2018 e trata-se do único serviço de enfermagem especializado em Podiatria Clínica em uma unidade pública de saúde no país. Inicialmente, seu funcionamento (2018) ocorreu durante as segundas-feiras das 08:00 às 17:00 horas, contando com a carga horária de dois docentes da faculdade de enfermagem, coordenadores dos projetos de extensão, que são enfermeiros habilitados e especializados na área de Podiatria Clínica, além das capacitações complementares em laserterapia, ozonioterapia e podoposturologia. Houve a realização de 337 consultas de enfermagem podiátrica aos pacientes diabéticos e capacitação por meio do Curso de Habilitação em Podiatria Clínica de 16 enfermeiros.

Em 2019, devido à grande demanda clínica dos pacientes, houve ampliação da oferta de atendimentos, para duas vezes semanais, mantendo-se o horário das 08:00 às 17:00 horas. Realizou-se 557 consultas de enfermagem em Podiatria Clínica, com média mensal de 47 atendimentos ao mês. Em relação a capacitação profissional habilitou-se 22 enfermeiros, sendo uma profissional, funcionária efetiva da instituição.

Em 2020, deu-se continuidade a oferta de atendimento duas vezes na semana, nos horários habituais, porém em articulações com a Coordenação de Enfermagem do complexo ambulatorial, conquistou-se a sessão da carga horária da enfermeira habilitada em Podiatria Clínica para atuação serviço, possibilitando assim, a ampliação de oferta de atendimento de 
duas para três vezes por semana para o ano de 2021. Realizou-se 515 consultas em 2020 e capacitação de 26 enfermeiros através do curso de habilitação.

Em vigência da pandemia Covid-19 para prevenção da disseminação da doença e proteção dos pacientes atendidos no serviço de Podiatria Clínica que fazem parte do grupo de risco por serem diabéticos e apresentarem comumente outras comorbidades associadas, ocorreu a implementação do monitoramento telefônico e da tele consulta de enfermagem entre os meses de março a julho de 2020. Obteve-se assim redução de $80 \%$ dos atendimentos presenciais e manutenção apenas de $20 \%$ atendimentos presenciais de casos graves tais como: paciente em tratamento da úlcera do pé diabético com infecção e/ou comprometimento osteoarticular (osteomielite), Neuroartropatia de Charcot, complicações da Doença Arterial Periférica (DAP) e emergências podiátricas como a onicocriptose (unha encravada) que pode levar a quadros infecciosos e até mesmo amputação em pacientes diabéticos.

Neste contexto, realizou-se monitoramento telefônico e a teleconsulta de enfermagem com vistas a informar aos pacientes sobre a importância de manutenção do isolamento social e todas as medidas de prevenção sanitária já que compunham grupo de risco da Covid-19.

Cabe registrar que durante as consultas presenciais de enfermagem especializadas em Podiatria Clínica são realizados os rastreios da neuropatia periférica através da avaliação neurológica não invasiva, como também, da avaliação da doença arterial, por meio da avaliação vascular e realização do índice tornozelo braquial.

Dentre as principais intervenções realizadas nas consultas podiátricas cita-se: podoprofilaxia, espiculectomia, aplicação de onicoórtese e órteses de proteção, laserterapia, ozonioterapia, curativos grau II, além de avaliação podopostural, realização de plantigrafia e podoscopia dos pés dos pacientes para confecção de palmilhas posturais sob molde e encaminhamentos para centros de reabilitação para aquisição de próteses de membros inferiores, órteses e calçados terapêuticos e/ou ortopédicos.

Sobre as características dos pacientes em acompanhamento todos têm diagnóstico médico de diabetes, podendo ser tipo I, tipo II ou gestacional. Além do diabetes as principais comorbidades associadas são a hipertensão arterial sistêmica, obesidade e dislipidemia.

Em relação as principais alterações podais e distrofias ungueais encontram-se a neuropatia periférica, doença arterial periférica, úlceras de pé diabético, neuroartropatia de charcot, hálux valgus, dedos em garra, hiperqueratose e calosidades, fissuras plantares, onicomicose, tínea pedis, onicocriptose e onicofose.

Em relação as contribuições técnicas na área de Podiatria Clínica, elaborou-se ficha de anamnese e exame clínico para acompanhamento do paciente com diabetes no serviço de Podiatria Clínica, folder educativo, protocolos para a utilização da laserterapia, materiais didáticos e treinamento em serviço para a equipe multidisciplinar. Ademais, promoveu-se anualmente o Seminário de Enfermagem em Podiatria Clínica no Estado do Rio de Janeiro e Curso de Habilitação para enfermeiros (2018, 2019, 2020, 2021) na universidade para difusão do conhecimento e capacitação de profissionais com interesse na área, além de palestra em Congresso Internacional relatando a importância do cuidado de enfermagem especializado em Podiatria Clínica para aos pacientes diabéticos e relatando a experiência do serviço no ambulatório em questão.

\section{Discussão}

O Diabetes Mellitus (DM) consiste em um distúrbio metabólico caracterizado por hiperglicemia persistente, decorrente de deficiência na produção de insulina ou na sua ação, ou em ambos os mecanismos ${ }^{3}$. Atinge proporções epidêmicas, com estimativa de 425 milhões de pessoas com DM no mundo. A hiperglicemia persistente está associada a complicações crônicas micro e macrovasculares, aumento de morbidade, redução da qualidade de vida e elevação da taxa de 
mortalidade. A classificação do DM baseia-se em sua etiologia e os fatores causais dos principais tipos de DM - genéticos, biológicos e ambientais ainda não são completamente conhecidos ${ }^{3}$.

De acordo com fonte de dados internacionais, em 2017, estimou-se que 8,8\% (intervalo de confiança de 95\%: 7,2 a 11,3) da população mundial com 20 a 79 anos de idade (424,9 milhões de pessoas) vivia com diabetes. Se as tendências atuais persistirem, o número de pessoas com diabetes foi projetado para ser superior a 628,6 milhões em $2045^{1}$.

Embora sejam muitas as complicações graves e dispendiosas que afetam os indivíduos com diabetes, tais como doenças cardiovasculares, problemas renais e retinopatia, as complicações com os pés representam a maior parte dessas complicações e são responsáveis por 40 a 70\% de todas as amputações das extremidades inferiores relacionadas ao diabetes ${ }^{2}$. Neste particular, o profissional de saúde tem papel fundamental na realização de práticas que aliem educação em saúde, treinamento e sistematização do autocuidado ${ }^{13}$.

A Podiatria Clínica surge da necessidade de ofertar cuidados especializados, seguros e de qualidade, utilizando-se das inovações tecnológicas disponíveis e atuando de forma autônoma e em consonância com os avanços e descobertas científicas por meio da sistematização da assistência de enfermagem.

A sistematização por meio da consulta de enfermagem, atividade privativa do enfermeiro ${ }^{14}$, tem como principal foco de identificar com alta acuidade as pessoas com DM que apresentam riscos de complicações. Desta forma, a avaliação minuciosa dos membros inferiores constitui etapa fundamental no rastreamento do risco de complicações nos pés. Ao realizar a consulta, o enfermeiro deve procurar identificar por meio de rigorosa inspeção e palpação, as alterações dermatológicas, musculoesqueléticas, vasculares e neurológicas. Esta avaliação criteriosa dos membros inferiores deve ser feita com a finalidade de identificar os riscos de futuras complicações, como por exemplo as ulcerações, e desta forma trabalhar a prevenção dos mesmos ${ }^{15}$.

Ressalta-se que para que o enfermeiro seja capaz de identificar com propriedade os sinais e sintomas, assim como as evidências presentes em exames complementares que indiquem a presença de riscos ou vulnerabilidades ao desenvolvimento de complicações, faz-se necessário formação e o desenvolvimento de habilidades e competências específicas no âmbito da Podiatria Clínica.

Em relação aos fatores de risco para o desenvolvimento de ulcerações os mais prevalentes foram: o corte inadequado das unhas, a utilização de calçados inapropriados, presença de micoses, calosidades, rachaduras e ressecamento da pele. Também foram associados o enchimento capilar inadequado, a perda de sensibilidade e o histórico de úlceras prévias ${ }^{16}$.

Vale ressaltar a importância da avaliação especializada em caso de lesões, como a úlcera de pé diabético, para escolha de um tratamento efetivo, sistematizado, individualizado e eficaz, o que reduz a gravidade de complicações, como amputações e comprometimento da qualidade de vida dos pacientes ${ }^{14}$. Recomenda-se o uso de ferramentas validadas para essa finalidade, ou seja, a prática baseada em evidências, com vistas a qualidade do cuidado de enfermagem.

Após o exame minucioso dos pés deve ser realizada a classificação do risco detectado, por meio de escores, a qual abrange desde o baixo risco até o risco mais elevado. Além de apontar o escore de risco para ulcerações, o sistema de classificação de risco permite definir a periodicidade de acompanhamento e avaliação do paciente ${ }^{14,15}$. O ato contínuo ao exame para avaliação dos pés, envolve uma elaboração de planejamento ou protocolo para o tratamento adequado das lesões já instaladas, pois o risco de amputações neste quadro clínico é maior e afeta diretamente a qualidade de vida dos pacientes ${ }^{13,15,17}$.

Reconhecer precocemente os fatores de risco do problema por meio da avaliação clínica, identificando a gravidade e distribuição da perda sensorial nos pacientes com DM, favorece o planejamento e implementação de intervenções baseadas em evidências, com foco na prevenção de danos sensoriais e tratamento do problema com vistas à qualidade de vida do indivíduo ${ }^{18}$. 
Uma das avaliações que podem ser realizadas pelo enfermeiro dentro da consulta de enfermagem é a avaliação neurológica não invasiva, que objetiva detectar o pé em risco e a neuropatia periférica. O principal instrumento utilizado para avaliação de risco é o monofilamento de Semmes Weinstein de 10g, utilizado para identificar o limiar de percepção cutânea. Além disso, realiza-se a avaliação das fibras finas (não mielinizadas) através da sensibilidade tátil, dolorosa, temperatura, e das fibras grossas (mielinizadas) além da sensibilidade vibratória e do reflexo do tendão calcâneo. Esse exame tem intuito de detectar alterações neurológicas, com o foco a identificação precoce da neuropatia periférica e o risco de complicações futuras tais como as alterações: sensitivas, motoras e autonômicas que elevam o risco de aparecimento da úlcera de pé diabético ${ }^{15-18}$.

Dando continuidade as avaliações, segue-se para a parte vascular, através da avaliação coloração e aspecto da pele, palpação dos pulsos (femoral, poplíteo, pedioso e tibial posterior) utilização do doppler vascular portátil para realização do Índice Tornozelo-Braquial (ITB). O ITB alterado pode ser um importante marcador de risco cardiovascular e de vasculopatias periféricas, pois a observação da alteração dos valores apresentados no paciente diabético contribui para determinar ou evidenciar maior probabilidade de aparecimento da Doença Arterial Obstrutiva Periférica (DAOP) que interfere na perfusão do membro afetado e consequentemente na cicatrização das úlceras de pé diabético e no aumento dos índices de amputação ${ }^{19}$.

Tão importante quanto as avaliações e estratégias de prevenção encontram-se as ações voltadas ao tratamento ou manejo clínico das complicações já existentes, como também, a reabilitação do paciente diabético. Neste sentido, o uso das tecnologias em saúde como ferramentas do cuidado de enfermagem, têm se mostrado um importante recurso terapêutico.

No âmbito das tecnologias, destaca-se a laserterapia de baixa intensidade. A terapia com laser de baixa intensidade é um recurso terapêutico de baixo custo e eficiência comprovada no tratamento de feridas, como é o caso das úlceras do pé diabético, pois tem a capacidade de acelerar o processo de reparo tecidual por meio do emprego de fontes de luz de baixa potência, como diodos emissores de luz (Light Emitting Diode - LED) ${ }^{19}$. Na atualidade sabe-se que a laserterapia de baixa intensidade tem se mostrado um dos recursos terapêuticos que vem sendo amplamente utilizado na prática clínica e apresenta uma gama de efeitos em tecidos vivos, tais como: melhoria da qualidade da cicatrização, estímulo a microcirculação, efeito antiinflamatório, antiedematoso e analgésico. Esse recurso pode ser utilizado pelo enfermeiro com capacitação na área. Estudo demonstra que a laserterapia de baixa intensidade realizada tanto isoladamente quanto associada ao óleo de calêndula officinalis, demonstra eficácia no alívio da dor e na aceleração do processo de reparo tecidual da úlcera do pé diabético, podendo ainda ser utilizada combinada a terapias alternativas ou coberturas diversas para o tratamento das lesões ${ }^{19}$.

No âmbito da avaliação da biomecânica e utilização de calçados especiais, observou-se um dado relevante no contexto de comportamentos de risco relacionado ao desconhecimento ou em não realizar o cuidado referente aos pés. Dados de pesquisa demonstram que $23,1 \%$ de seus participantes não fazem exame dos pés e 46,2\% realizam a retirada de cutículas. Trata-se de uma precaução referida na literatura em muitas pesquisas sobre o conhecimento de boas práticas de cuidados com os pés, as quais incluem: inspeção diária; realização de exercícios para os pés; não andar descalço; uso de sapatos adequados, macios e confortáveis; verificação do espaço interno dos sapatos antes de usá-los; uso de meias de algodão sem costura; corte adequado das unhas; não utilizar bolsa de água quente ou escalda-pés; secar os espaços interdigitais ${ }^{13}$.

Como implicações para a prática clínica, entende-se que a identificação dos fatores de risco por meio do presente estudo subsidia a atuação clínica do enfermeiro no atendimento específico para esta população e cria a necessidade da implantação, nos serviços de saúde, de centros de referência com especificidade da Enfermagem em Podiatria Clínica, bem como de enfermeiros treinados e capacitados para o acompanhamento do paciente diabético, que requer um cuidado com muitas especificidades em sua prática e desenvolvimento de ações destinadas ao rastreamento precoce e prevenção de complicações do DM, assim como a educação dos pacientes para o autocuidado, tornando-os ativo nesse processo ${ }^{17}$. 


\section{Conclusão}

Considerando que o serviço de enfermagem em Podiatria Clínica reúne conhecimentos e técnicas especializadas que permitem a avaliação dos pés para a identificação de indícios de risco que possam comprometer a habilidade funcional dos indivíduos. Sendo assim, contribui para a redução de complicações e possíveis amputações nos pacientes com patologias nos membros inferiores em decorrência do diabetes.

Desse modo, a oferta de cuidados especializados de enfermagem, por meio do serviço de Podiatria Clínica, atrelada ao uso de tecnologias como a laserterapia de baixa intensidade e a ozonioterapia, consistem em importante ferramenta para avaliação, prevenção, tratamento e reabilitação dos pacientes, além de atividades inclusivas no âmbito do SUS através de práticas avançadas de enfermagem para o cuidado das patologias dos membros inferiores que favorecem a melhoria da qualidade de vida da população assistida.

O curso de Habilitação em Podiatria Clínica promove um resgate e adequação de conhecimento técnico-científico a atual formação do Enfermeiro, fornecendo habilidades e competências a este profissional para a prestação de assistência em Podiatria. Esses conteúdos são necessários ao ensino de graduação de forma generalista no que tange a avaliação neurológica não invasiva, muito importante para o estudante e futuro profissional. E de forma mais abrangente e especializada por meio dos cursos de pós-graduação lato sensu, Especialização em Podiatria Clínica. Além disso, a expansão dessa especialidade e formação de novos profissionais na área, bem como, os atendimentos especializados prestados à clientela através dessa proposta de extensão, irão fomentar estudos e dar subsídios à criação efortalecimento de pesquisas na área da Enfermagem em Podiatria Clínica, ainda muito escassa no Brasil, além de divulgar e difundir a especialidade por todo país.

\section{Referências}

International Diabetes Federation (2017). Clinical Practice Recommendation on the Diabetic Foot: a guide for health care professionals: International Diabetes Federation.

Brasil. Ministério da Saúde (2016). Secretaria de Atenção à Saúde. Manual do pé diabético: estratégias para o cuidado da pessoa com doença crônica. Diário Oficial da União. Brasília.

Sociedade Brasileira de Diabetes (2021). Diretrizes da Sociedade Brasileira de Diabetes. Editora Clannad. Brasília, 2019-2020. www.diabetes.gov.br.

Batista, F (2010). Uma abordagem multidiciplinar sobre o pé diabético. Andreoli.

Avner, S. N., Baruch, K., \& Henri, T (2006). Two novel itraconazole pulse therapies for onychomycosis: a 2-year follow-up. J Dermatolog Treat., 17 (2): 11720. $10.1080 / 09546630600621953$.

Portal do Enfermeiro Podiatra Brasileiro (2021). A história da podiatria. http://www.podiatria.com.br/index/historia

Brasil. Decreto $\mathrm{n}^{\circ} 10.068$ de 23 de março de 1939 (1939). Regula a forma de habilitação dos enfermeiros, em geral, e dá outras providências. Assembleia Legislativa do Estado de São Paulo.

Minayo, M. C. S., \& Deslandes, S. F (2015). Pesquisa social: teoria, método e criatividade. (34a ed.), Vozes.

Trentino, M., Paim, L., \& Silva, D. M. G. V (2017). O método da pesquisa convergente assistencial e sua aplicação na prática de enfermagem. Texto $e$ Contexto Enferm, 25 (4): 1-10. https://doi.org/10.1590/0104-07072017001450017.

Breda, K. M. L(2015). What is old is also new - participatory action research. Texto Contexto Enferm, 24 (1): 9-10. http://dx.doi.org/10.1590/0104$07072015000 \mathrm{EDIT} 01$.

Policlínica Piquet Carneiro (2021). Mapeamento e análise dos serviços e estruturas organizacionais da Policlínica Piquet Carneiro. http://www.ppc.uerj.br

Brasil. Ministério da Saúde (2012). Resolução $n^{\circ}$ 466, de 12 de dezembro de 2012. Aprova diretrizes e normas regulamentadoras de pesquisas envolvendo seres humanos. Brasília, Diário Oficial da União, 12 dez.

Santana, S. L. W., Souza, S. J., Rossi, S. C. F., et al., (2016). Promoção da saúde de pessoas com diabetes mellitus no cuidado educativo preventivo do pé diabético. Cienc. enferm. 22 (2): 103-116. https://scielo.conicyt.cl/pdf/cienf/v22n2/art_08.pdf.

Brasil (2017). Resolução do Conselho Federal de Enfermagem n ${ }^{\circ} 544$ de 9 de maio de 2017. Dispõe sobre a Consulta de Enfermagem. Distrito Federal.

Padilha, A. P., Rosa, L.M., Schoeller, S. D., et al., (2017). Manual de cuidado às pessoas com diabetes e pé diabético: construção por scoping stusy. Texto contexto - enferm. 26 (4): 2-11. http://dx.doi.org/10.1590/0104-07072017002190017. 
Research, Society and Development, v. 10, n. 6, e2710615353, 2021

(CC BY 4.0) | ISSN 2525-3409 | DOI: http://dx.doi.org/10.33448/rsd-v10i6.15353

Teston, E., Senteio, J., Ribeiro, B., et al., (2017). Fatores de risco para ulceração no pé de indivíduos com diabetes mellitus tipo 2. Cogitare Enfermagem. 22 (4): 1-9. http://dx.doi.org/10.5380/ce.v22i4.51508.

Lucoveis, M. L. S., Gamba, M. A., Boccara A., et al., (2018). Grau de risco para úlceras nos pés por diabetes: avaliação de enfermagem. Rev. Bras. Enferm. 71 (6): 3041-3047. https://doi.org/10.1590/0034-7167-2017-0189.

Noronha, J. A. F (2019). Fatores associados à alteração da percepção sensorial tátil nos pés de pacientes com diabetes mellitus: Tese (Doutorado). Universidade Federal de Minas Gerais, Escola de Enfermagem. $176 \mathrm{f}$.

Carvalho, A. F. M., Feitosa, M. C. P., Coelho, N. P. M. F., et al., (2016). Low-level laser therapy and Calendula officinalis in repairing diabetic foot ulcers. Rev. esc. enferm. USP. 50 (4): 628-634. http://dx.doi.org/10.1590/S0080-623420160000500013. 